\title{
DEADLOCK AVOIDANCE TN GRAPH-STRUCTURED TASK SYSTEMS
}

by

\author{
M.Di MANZO, A.I. Erisiani \\ Istituto di Elettrotecnica \\ Università di Genova, Italy
}

and

G. O1impo

Laboratorio per le Tecnologie Didattiche

Consiglio Nazionale Ricerche, Genova, Italy

\section{SUMMARY}

Existing models for deadlock detection and avoidance give practical solution only in the case of chains of independent tasks.

In this paper we propose a non enumerative approach to deadlock avoidance when the workload consists of a graph-structured task system. The avoidance algorithm is based on an extention of the Coffman and Denning deadlock model.

\section{Introduction}

The problem of deadlock avoidance has been widely examined by several authors $[2,4,5,7]$. However, most researches have been concerned mainly with systems of independent processes, and only a few results are known for the case of interacting processes [5]. Moreover, the deadlock detection and avoidance methods which have been detined for graph-structured task systems are essentially enumerative, and therefore quite time consuming; for practical implementation purposes sufficient and rapidly verifiable conditions are needed. On the other hand, the extensive development of parallel processing techniques and multiprocessor architecture is increasing the interest in process cooperation as a very basic tool for programing methodologies and computer systems operations: so, we feel that deadlock avoidance techniques for large systems of interacting processes should be improved.

The aim of this paper is to present a practical method for deadlock avoidance when the workload is a system of interacting processes that can be structured as a graph of tasks. With respect to the basic assumptions our model is quite close to that developed by Coffman and Deming [2]; we made this choice because the mode1 in [2] is perhaps the most widely known and the most suitable for the extension to the case of our interest. Therefore in the following we will often refer to [2], even if a number of fundamental definitions are reported here in order to allow a self-contained reading of the paper. The paper is organized as follows. In section 2 a set of basic definitions is presented, and a theorem is proved which allows the detection of deadlock in a general graph structured task system; in section 3 we discuss the problem of deadlock avoidance in the simplified case of a tree structured task system; in section 4 an approach to deadlock avoidance with graph structured task systems is suggested. 


\section{Basic definitions and theorem}

We define a task system to be a pair $G=(I, \infty)$, where $I=T_{1}, T_{2}, \ldots, T_{n}$ is a set of tasks, and $<$ is a partial ordering (precedence relation) on $I$. Given two tasks $T$ and $T^{\prime}, T<T^{\prime}$ means that task $T$ is to be completed before task $T^{\prime}$ begins. A task system can be represented by a precedence graph, where each vertex is a task and the vertices corresponding to two tasks $T$ and $T^{\prime}$ are, connected by a directed edge iff $T \ll T^{\prime}$. A path of length $k$ through the precedence graph $G$ is a sequence of edges $\left(\mathrm{T}_{\mathrm{r}_{1}} \mathrm{~T}_{\mathrm{r}_{2}}\right)$ $\left(\mathrm{T}_{\mathrm{r}_{2}} \mathrm{~T}_{\mathrm{r}_{3}}\right) \ldots\left(\mathrm{T}_{\mathrm{r}_{\mathrm{k}-1}} \mathrm{~T}_{\mathrm{r}_{\mathrm{k}}}\right)$ passing thorugh vertices $\mathrm{T}_{\mathrm{r}_{1}}, \mathrm{~T}_{\mathrm{r}_{2}}, \ldots, \mathrm{T}_{\mathrm{r}_{\mathrm{k}}}$. For $i$ and $j$ such that $1 \leqslant i<j \leqslant k, T_{r_{j}}$ is a predecessor of $T_{r_{j}}$ and $T_{r_{j}}$ is a successor of $\mathrm{T}_{\mathrm{r}_{i}}$; if $j=i+1$ then $\mathrm{T}_{\mathrm{r}_{i}}$ is an immediate predecessor of $\mathrm{T}_{r_{j}}$ and $\mathrm{T}_{\mathrm{r}_{j}}$ is an immediate successor of $T_{r_{i}}$. Let $S_{i}$ be the set of immediate successors of $T_{i}$ and $P_{i}$ be the set of immediate predecessors of $\mathrm{T}_{i}$. A task with no predecessors is an initial task and a task with no successors is a terminal task. The level of task $T_{i}$ (written $\ell\left(T_{i}\right)$ ) is $k$ if the length of the longest path from $T_{i}$ to any terminal task is $k$. An execution sequence of a system $G$ of $n$ tasks is a sequence of task initiations and terminations $\alpha=a_{1} a_{2} \cdots a_{2 n}$ satisfying the following conditions:

1. For every task $T \in I$ the symbols $\bar{T}$ (task initiation) and $I$ (task termination) appear exactly once in $\alpha$.

2. If $a_{i}=\bar{T}$ and $a_{j}=T$, then $i<j$.

3. If $a_{i}=\underline{T}$ and $a_{j}=\bar{T}$, and $T<T^{\dagger}$, then $i<j$.

More details can be found in [2].

Since a task represents a computation unit during which total resource requirements don't change, the only significant events are task initiations and terminations. If the physical system consists of $m$ resource types ${ }^{*}$, it is possible to define for each task $\mathrm{T}_{i}$ a request vector ${ }^{* *} \hat{q}_{i}=\left(\mathrm{q}_{i 1}, \mathrm{q}_{i 2}, \ldots, \mathrm{q}_{i m}\right)$, representing the number of units of each resource type which must be allocated to the task $T_{i}$ before its initiation, a release vector $\hat{r}_{i}=\left(r_{i 1}, r_{i 2}, \ldots, r_{i m}\right)$, representing the number of units of each resource type which are released to the system by task $T_{i}$ on its termination, and a transfer vector $\hat{t}_{i j}=\left(t_{i j 1}, t_{i j 2}, \ldots, t_{i j m}\right)$ for each $t_{j} \in S_{i}$, representing the number of units of each resource type which are transferred to task $\mathrm{T}_{i}$ by task $\mathrm{T}_{i}$ on its termination. We suppose that every terminated task holds no unit of resource, and hence the following relation must be satisfied, for $1 \leqslant j \leqslant n$ :

$$
\hat{q}_{j}+\frac{\sum_{\forall: T_{i} \in P_{j}}}{\hat{t}_{i j}}=\hat{r}_{j}+\sum_{\forall k: T_{k} \in S_{j}} \hat{t}_{j k}
$$

\footnotetext{
* Obviously we will consider only those resource types which satisfy all necessary conditions for deadlock [2].

** From here on, vectors will be distinguished by the symbol $\wedge$.
} 
Given an execution sequence $\alpha=a_{1} a_{2} \ldots a_{n}$, a corresponding state sequence $\sigma=s_{0} s_{1} s_{2} \cdots s_{2 n}$ can be defined, each state $s_{k}$ specifying the amount of available resources and the number of resources units allocated to and requested by each task after the event $a_{k}$. Hence, each state $s_{k}$ is defined by $2 n+1$ vectors $\hat{v}(k), \hat{p}_{i}(k)$ and $\hat{Q}_{i}(k), \quad 1 \leqslant k \leqslant n$, where vector $\hat{v}(k)$ specifies the number of available units of each resource type, and vectors $\hat{p}_{i}(k)$ and $\hat{Q}_{i}(k)$ specify respectively the number of units of each resource type hold and requested by task $T_{i}$ after the event $a_{k}$. Vectors $\hat{\mathrm{P}}_{i}(\mathrm{k})$ and $\hat{\mathrm{Q}}_{\mathrm{i}}(\mathrm{k})$ are defined as follows ${ }^{*}$ :

1. $\hat{\mathrm{P}}_{i}(k)=0$ if $\exists_{j}:\left(a_{j}=\underline{T}_{i}\right) \wedge(j \leqslant k)$

2. $\hat{\mathrm{P}}_{i}(k)=\sum_{\ell \ell: \mathrm{T}_{\ell} \in P_{i}^{*}} \hat{\mathrm{t}}_{\ell i}$ if $\not j:\left(a_{j}=\overline{\mathrm{T}}_{i}\right) \wedge(j \leqslant k)$

where $P_{i}^{*}$ is the subset of $P_{i}$ such that $T_{\ell} \in P_{i}^{*}$ iff

$\mathrm{T}_{\ell} \in P_{i}$ and $\exists \mathrm{h}:\left(\mathrm{a}_{\mathrm{h}}=\mathrm{T}_{\ell}\right) \wedge(\mathrm{h} \leqslant \mathrm{k})$.

3. $\hat{\mathrm{P}}_{i}(\mathrm{k})=\sum_{\forall \ell: \mathrm{T}_{\ell} \in P_{i}} \hat{\mathrm{t}}_{\ell i}+\hat{\mathrm{q}}_{i}$ if $\exists j:\left(a_{j}=\overline{\mathrm{I}}_{i}\right) \wedge(j \leqslant k)$ and

$\not x_{\mathrm{h}}:\left(\mathrm{a}_{\mathrm{h}}=\mathrm{T}_{\mathrm{i}}\right) \wedge(\mathrm{h} \leqslant \mathrm{k})$.

4. $\hat{Q}_{i}(k)=\hat{q}_{i}$ if $\exists h:\left(a_{n}=T_{j}\right) \wedge(h \leqslant k)$ for each $T_{j} \in P_{i}$ and

fle: $\left(\mathrm{a}_{\ell}=\overline{\mathrm{T}}_{\mathrm{j}}\right) \wedge(\ell \leqslant \mathrm{k})$.

5. $\hat{Q}_{\mathbf{i}}(\mathrm{k})=0$ otherwise.

In the initial state $s_{0}$ :

1. $\hat{v}(0)=\hat{R}$, the vector specifying the total amount of system resources.

2. $\hat{\mathrm{P}}_{i}(0)=0$ for $1 \leqslant i \leqslant n$.

3. $\hat{Q}_{i}(0)=q_{i}$ if $T_{i}$ is an initial task, $\hat{Q}_{i}(0)=0$ otherwise.

Given an execution sequence $\alpha=a_{1} a_{2} \ldots a_{k} \ldots a_{2 n}$, event $a_{k}=\bar{T}_{i}$ is allowable if $\hat{\mathrm{Q}}_{\mathbf{i}}(\mathrm{k}-1) \leqslant \hat{v}(\mathrm{k}-1)$. Obviously task terminations are always allowable events. An execution sequence is valid if all initiation events are allowable.

We give now a very intuitive definition of deadlock:

definition 1: Let $G$ be a system of $n$ tasks, $\alpha=a_{1} a_{2} \ldots a_{k}$ a partial valid execution sequence, $I$ a set of tasks such that $T_{i} \in I$ iff $\hat{Q}_{i}(k)>0$. State $s_{k}$ contains a deadlock if there is at least one task $T_{i} \in I$ such that it is impossible to find a valid partia1 execution sequence $\alpha^{\prime}=a_{1} a_{2} \ldots a_{k} a_{k+1} \ldots a_{p}$ defined as follows:

* From here on we define a vector $\hat{v}=\left(v_{1}, v_{2}, \ldots, v_{j}\right)$ to be a null vector $(\hat{v}=0)$ if $v_{i}=0$ for $1 \leqslant i \leqslant j ;$ a pesitive vector $(\hat{v}>0)$ is a not null vector such that $v_{i}>0$ for $1 \leqslant i \leqslant j$; a negative vector $(\hat{v}<0)$ is a not null vector such that $v_{i} \leqslant 0$ for $1 \leqslant i \leqslant j$. 


$$
\begin{aligned}
& \text { 1. } \alpha \text { is prefix of } \alpha^{\prime} \\
& \text { 2. } a_{p}=\bar{T}_{i}
\end{aligned}
$$

Task $\mathrm{T}_{\boldsymbol{i}}$ is then said to be deadlocked.

Definition 1 says that the system contains a deadlock if we are unable to initiate a task which is ready to be executed, all its predecessors being terminated. clearly, if a task is deadlocked, all its successors are also blocked, and a whole subgraph cannot be executed.

A deadlock detection procedure based directly on definition 1 is necessarily enumerative. Therefore, we must look for necessary and sufficient conditions which can be more easily verified. Such conditions are stated in the following theorem:

Theorem 1: Let $G$ be a system of $n$ tasks and $\alpha=a_{1} a_{2} \ldots a_{k}$ a partial valid execution sequence. Suppose that there is a non empty set $D$ of indices such that for each $i$ in $D$ :

$$
\hat{Q}_{i}(k) \notin \hat{v}(k)+\sum_{j \notin D \times D^{*}} \hat{p}_{j}(k)
$$

where $D^{*}$ is the set of indices such that $\ell \in D^{*}$ iff $T_{\ell}$ is a successor of $T_{i}$, for any $i \in D$. Then $s_{k}$ contains a deadlock and every task $T_{i}, i \in D$, is deadlocked.

Theorem 1 is an obvious generalization of Denning's definition, but its proof is quite cumbersome and so it is not discussed here (see Appendix). This theorem is interesting mainly because it proves that the definition of deadlock given in [2] can be slightly tnodified to cover the case of graph structured task systems and gives a simple method to detect deadlock situations. However, our specific goal is avoidance rather than detection, and to avold deadlock each state must be checked for safeness, according to the following definitions:

Definition 2: Let $\alpha=a_{1} a_{2} \ldots a_{k}$ be a partial valid execution sequence: state $s_{k}$ is safe if there exists at least one valid complete execution sequence having $\alpha$ as a prefix.

In the following sections a further step will be made, looking for sufficient conditions for safeness. We are restricting ourselves to sufficient conditions because we are unable to find necessary and sufficient conditions that are not essentially enumerative.

\section{Tree structured task systems}

We will consider at first a special case, that of a tree structured task system. This model can represent a situation in which a process, at a particular stage of its activi- 
ty, starts the execution on one or more processes, which run independently of each other, starting in turn new sets of processes and so on.

A sufficient condition for safeness can be based on condition (2.2). In fact, suppose that if task $T_{i}$ can be initiated, then the whole subtree $G_{i}$, having $T_{i}$ as root, can be terminated; this means that there is at least one partial execution sequence, involving the whole set of tasks of subtree $G_{i}$, that is valid if the resources granted to $T_{i}$ become available to its successors. In such a case, if no task asking for resources is deadlocked, then the system is in a safe state. These remarks may be formalized as follows:

Theorem 2: Let $G$ be a tree structured task system, $\alpha=a_{1} a_{2} \ldots a_{k}$ a partial valid execution sequence and $I$ a set of indices such that $i \in I$ iff the immediate predecessor of $T_{i}$ is terminated but $\underline{T}_{i} \notin \alpha$. Each task $T_{i}$ is the root of a subtree $G_{i}$. Let $\hat{M}_{i}^{*}$ be a vector representing the minimal amount of resources that must be allocated to subtree $G_{i}$ to guarantee that all tasks belonging to $G_{i}$ can be terminated. Let $\hat{Q}_{i}^{*}(k)=\hat{M}_{i}^{*}-\hat{P}_{i}(k)$, for each $i \in I$. If no set of indices $D \in I$ can be found, such that, for each $i \in D$ :

$$
\hat{Q}_{i}^{*} * \hat{v}(k)+\sum_{j \notin D} \hat{P}_{j}(k)
$$

then state $s_{k}$ is safe.

Proof. If no set $D$ exists satisfying (3.I), then at least one index $i_{1} \in I$ exists, such that $\hat{Q}_{\dot{i}_{1}}^{*} \leqslant \hat{v}(k)$ (otherwise a set $D=I$ would satisfy (3.1)). This means that the available resources suffice to complete subtree $G_{i_{1}}$. On completion, $G_{i 1}$ release all allocates resources, which are $\hat{\mathrm{P}}_{i_{1}}(\mathrm{k})$ plus all subsequently obtained units up to the maximum $\hat{M}_{i_{I}}^{*}$. Therefore, the amount of available resources is now $\hat{v}(k)+\hat{P}_{i_{1}}(k)$, but, by hypothesis, at least one index $i_{2} \in I$ exists such that $\hat{Q}_{i_{2}}^{*} \leqslant \hat{v}(k)+\hat{p}_{i_{1}}(k)$; so subtree $G_{i_{2}}$ can be completed and so on.

Condition (3.1) is only sufficient, as can be proved by a simple counter-example. Consider the following system:

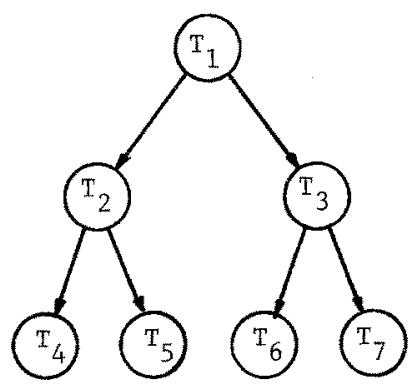

$$
\begin{aligned}
& \hat{\mathrm{R}}=(3,3) \\
& \hat{\mathrm{q}}_{1}=(2,2), \hat{\mathrm{r}}_{1}=(0,0), \hat{\mathrm{t}}_{12}=\hat{\mathrm{t}}_{13}=(1,1) \\
& \hat{\mathrm{q}}_{2}=(1,1), \hat{\mathrm{r}}_{2}=(0,0), \hat{\mathrm{t}}_{24}=\hat{\mathrm{t}}_{25}=(1,1) \\
& \hat{\mathrm{q}}_{3}=(1,1), \hat{\mathrm{r}}_{3}=(2,1), \hat{\mathrm{t}}_{36}=(0,1), \hat{\mathrm{t}}_{37}=(0,0) \\
& \hat{\mathrm{q}}_{4}=(1,0), \hat{\mathrm{r}}_{4}=(2,1) \\
& \hat{\mathrm{q}}_{5}=(2,1), \hat{\mathrm{r}}_{5}=(3,2) \\
& \hat{\mathrm{q}}_{6}=(3,1), \hat{\mathrm{r}}_{6}=(3,2) \\
& \hat{\mathrm{q}}_{7}=(2,2), \hat{\mathrm{r}}_{7}=(2,2)
\end{aligned}
$$


Let $\alpha=\bar{T}_{1} I_{1}$ be the partial execution sequence. Then state $s_{2}$ is defined by:

1. $\hat{v}(2)=(1,1)$

2. $\hat{\mathrm{P}}_{2}(2)=\hat{\mathrm{P}}_{3}(2)=(1,1), \hat{\mathrm{P}}_{\mathrm{i}}(2)=(0,0)$ for $\mathrm{i} \notin\{2,3\}$

3. $\hat{Q}_{2}(2)=\hat{Q}_{3}(2)=(1,1), \hat{Q}_{i}(2)=(0,0)$ for $i \neq\{2,3\}$

4. $\hat{M}_{2}=\hat{M}_{3}=(3,2)$

5. $\hat{Q}_{2}(2)=\hat{Q}_{3}(2)=(2,1)$

It can be easily verified that the set of indices $D=\{2,3\}$ satisfies $(3,1)$ but the system is not deadlocked, because the sequence

$$
\alpha^{\prime}=\overline{\mathrm{T}}_{1} \underline{\mathrm{T}}_{1} \overline{\mathrm{T}}_{3} \underline{T}_{3} \overline{\mathrm{T}}_{2} \underline{\mathrm{T}}_{2} \overline{\mathrm{T}}_{4} \underline{\mathrm{T}}_{4} \overline{\mathrm{T}}_{5} \underline{\mathrm{T}}_{5} \overline{\mathrm{T}}_{6} \underline{\mathrm{T}}_{6} \overline{\mathrm{T}}_{7} \mathrm{~T}_{7}
$$

is a valid execution sequence.

The central point in theorem 2 is the evaluation of the vectors $\hat{M}^{*}$ s.

A truly minimal vector is quite difficult to define for two main reasons. First, it is not guaranteed that an unique minimal vector exists; in the above example we could define a different vector $\hat{\mathrm{M}}_{3}^{*}=(2,3)$, which is as minimal as $\hat{\mathrm{M}}_{3}^{*}$, because if two units of the first type and three of the second type were allocate to subtree $G_{3}$ all tasks of $G_{3}$ could be completed, and $\hat{M}_{3}^{*} \ngtr \hat{M}_{3}^{*}$ (as well as $\hat{M}_{3}^{*} \ngtr \hat{M}_{3}^{* \prime}$ ). Secondly, the problem of finding a vector $\hat{M}_{i}^{*}$ for subtree $G_{i}$ is essentially the same as sequencing all the rasks of a tree to verify if, for a given set of system resources, the initial state is safe. To be sure that a set of resources is minimal, we must prove that substracting even one unit of resource we make the initial state unsafe; this test could be performed in a non enumerative way if necessary conditions for safeness were known, but theorem 2 gives us only sufficient conditions. Therefore, to avoid enumerative methods, we will look for a minimal vector $\hat{M}_{i} \geqslant \hat{M}_{i}^{*}$, trying to define $\hat{M}_{i}$ as close as possible to $\hat{M}_{i}^{*}$. A recursive method to compute $\hat{M}_{i}{ }^{\prime} s$ can be based on the following theorem:

Theorem 3: Let $G$ a tree structured task system, $G_{i}$ a subtree having task $T_{i}$ as root, $\left\{G_{i_{1}}, G_{i_{2}}, \ldots, G_{\mathfrak{I}_{n}}\right\}$ the set of subtrees having as roots the immediate successors of $\mathrm{T}_{i}$, and $\mathrm{T}_{\mathrm{s}}$ the immediate predecessor of $\mathrm{T}_{i}$.

Let $I$ be the set of indices such that $j \in I$ iff $T_{j} \in S_{i}$. Let $\hat{v}_{i}$ be a vector of resources such that no set of indices DCI can be found having the following property:

$$
\hat{M}_{j}-\hat{t}_{i j} \leqslant \hat{v}_{i}+\sum_{k \neq D} \hat{t}_{i k} \quad \text { for each } j \in D
$$

Choose $\hat{v}_{i}$ such that no vector $\hat{v}_{i}<\hat{v}_{i}$ can be found which satisfies the above requirement. Then

$$
\hat{M}_{i}=\hat{v}_{i}+\hat{t}_{s i}+\hat{q}_{i}-\hat{r}_{i}
$$

The proof is trivial and will be omitted.

The computation of $\hat{M}_{i}$ can be performed only once for every subtree $G_{i}$ of $G$, because $\hat{M}_{i}$ is independent of the specific execution sequence, as stated by (3.3) (in fact, no 
reference is made to any state of the sequence $\sigma)$. To compute $\hat{\mathrm{M}}^{\prime} \mathrm{s}$ the best approach is to find the immediate predecessors of terminal tasks, each identifying a subtree, to evaluate the resource requirements of this first set of subtrees, and to go on in this manner reducing step by step the tree to its root.

\section{Graph structured task systems}

In the case of graph structured task systems theorem 2 cannot be applied because of the complexity of precedence relations; therefore a different approach to safeness verification is needed.

Informally, a criterion for safeness can be stated as follows. Given a task system $G$, suppose that a partial valid execution sequence $\alpha_{k}$ has been found, containing the terminations of a11 tasks at a level greater than $j$; suppose also that, if a11 tasks at a level $j$ are terminated, then a valid completion sequence can be found. Then the state $s_{k}$ is safe if no task at level $j$ is deadlocked. By recursion a general rule to verify safeness can be stated, which implies the possibility of executing the task system level by level.

Consider, for instance, the following task system

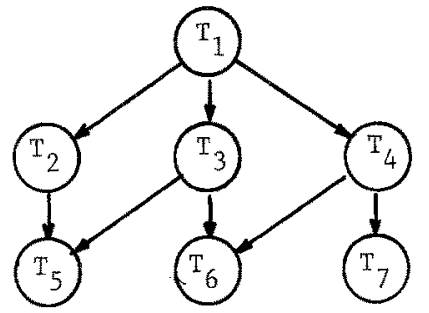

and suppose it can be executed level by level, that is the following execution sequence is valid:

$$
\alpha^{*}: \quad \overline{\mathrm{T}}_{1} \mathrm{~T}_{1} \overline{\mathrm{T}}_{2} \mathrm{I}_{2} \overline{\mathrm{T}}_{3} \underline{\mathrm{T}}_{3} \overline{\mathrm{T}}_{4} \underline{\mathrm{T}}_{4} \overline{\mathrm{T}}_{5} \mathrm{I}_{5} \overline{\mathrm{T}}_{6} \mathrm{~T}_{6} \overline{\mathrm{T}}_{7} \mathrm{~T}_{7}
$$

Consider now the partial execution sequence $\alpha: \bar{T}_{1} \mathrm{~T}_{1} \overline{\mathrm{T}}_{2} \underline{\mathrm{T}}_{2} \overline{\mathrm{T}}_{5}$; the activation of task $\overline{\mathrm{T}}_{5}$ does not lead to an unsafe state only if the amount of available resources after its termination allows us to execute tasks $\mathrm{T}_{3}$ and $\mathrm{T}_{4}$.

We will now fornalize the above criterion, proving at first the following lemma.

lemma 1: Let $G$ be a system of tasks, $\alpha=a_{1} a_{2} \ldots a_{k}$ a partial valid execution sequence, $I$ a set of indices such that $i \in I$ if $\hat{Q}_{i}(k)>0$. Suppose $\hat{q}_{i}-\hat{r}_{i} \geqslant 0$ for each $i \in I$. Let $I_{p} \subset I$ be the subset of I such that $i \in I_{p}$ iff $\hat{q}_{i}-\hat{r}_{i}<0$ and $I_{n} \subset I$ the subset of I such that $j \in I_{n}$ iff $\hat{q}_{j}-\hat{r}_{j} \geqslant 0$. If no set of indices $D_{p} \subset I_{p}$ exists such that, for 
each $i \in D_{p}$ :

$$
\hat{q}_{i} \notin \hat{v}(k)+\sum_{j \in I_{p}}\left(\hat{r}_{j}-\hat{q}_{j}\right)
$$

and no set of indices $D_{n} \subset I_{n}$ exists such that, for each $i \in D_{n}$ :

$$
\hat{r}_{i} \notin \hat{v}(k)+\sum_{j \in I_{p}}\left(\hat{r}_{j}-\hat{q}_{j}\right)+\sum_{j \in D_{n}}\left(\hat{r}_{j}-\hat{q}_{j}\right)
$$

then a partial valid completion sequence $\alpha_{1}$ exists, such that $\underline{T}_{i} \in \alpha_{1}$ for every $i \in I$.

Proof. If no set $D_{p}$ exists such that (4.1) is satisfied, there is at least one task $\mathrm{T}_{\mathrm{i}_{1}}, \mathrm{i}_{1} \in \mathrm{I}_{\mathrm{p}}$, such that $\hat{\mathrm{q}}_{\mathrm{i}_{1}} \leqslant \hat{v}(\mathrm{k})$; otherwise a set $\mathrm{D}_{\mathrm{p}} \equiv \mathrm{I}$ would exist. This means that task $\mathrm{T}_{\mathrm{i}_{1}}$ can be initiated with the currently available resources. Let $\mathrm{I}_{\mathrm{p}_{1}}$ be the subset of $I_{p}$ made of all indices $i \in I_{p}$ except $i_{1}$. No set $D_{p}=I_{p_{1}}$ exists such that (4.1) is satisfied; therefore there is at least one task $T_{i_{2}}, i_{2} \in I_{P_{1}}$, such that $q_{i_{2}} \leqslant v(k)+$ $-q_{i_{1}}+r_{i_{1}}$. But the quantity $\hat{v}(k)-\hat{q}_{i_{1}}+\hat{r}_{i_{1}}$ represents the amount of available resources after the cermination of $T_{i_{1}}$, and hence task $T_{i_{2}}$ can be initiated after the termination of $\mathrm{T}_{\mathrm{i}_{1}}$. Proceeding in this manner it is possible to prove that a valid partial execution sequence $\alpha_{2}=\bar{T}_{i_{1}} I_{i_{1}} \bar{T}_{i_{2}} T_{i} \ldots$ exists, such that $T_{i} \in \alpha_{2}$ for each $i \epsilon I_{p}$. After the execution of all tasks $T_{i}$, $i \in I_{p}$, the amount of available resources is

$$
\hat{v}^{i}=\hat{v}(k)+\sum_{j \in I_{p}}\left(\hat{r}_{j}-\hat{q}_{j}\right)>\hat{v}(k)
$$

The execution of each task $T_{i}$, $i \in I_{n}$, does not increase the available resources, being $\hat{r}_{i}-\hat{q}_{i} \leqslant 0$. If no set $D_{n}$ exists such that (4.2) is satisfied, there is at least one task $T_{\ell_{1}}$ such that

$$
\hat{q}_{\ell_{1}} \leqslant \hat{v}^{\prime}-\sum_{\substack{j \in I_{n} \\ j \neq \ell_{1}}}\left(\hat{q}_{j}-\hat{r}_{j}\right)
$$

otherwise a set $D_{n}=I_{n}$ would exist. Therefore task $T_{\ell}$ can be initiated with the resources which are available after the execution of all tasks $T_{\ell}, \ell \in I_{n_{1}}$, being $I_{n_{1}}=$ $=I_{n}-\left\{\ell_{1}\right\}$. But no set $D_{n_{1}} \equiv I_{n_{1}}$ exists, so that at the end a partial valid execution sequence $\alpha_{3}=\ldots \ldots \overline{\mathrm{T}}_{\ell_{2}} \mathrm{I}_{l_{2}} \overline{\mathrm{T}}_{1} \mathrm{~T}_{l_{1}}$ can be found, such that $\mathrm{T}_{\ell} \in \alpha_{3}$ for each $\ell \in \mathrm{I}_{\mathrm{n}}$. Therefore, $\alpha_{1}=\alpha_{2} \| \alpha_{3}$.

Conditions (4.1) and (4.2) are also necessary if the set of possible sequences $\alpha_{1}$ is limited to sequen ces containing no events $\bar{T}_{i}$ or $\underline{T}_{i}$ with $i \notin I$. The proof is trivial and will be omitted. Also the following corollary can be trivially proved:

corollary 1 . Let $G$ be a system of tasks, $\alpha=a_{1} a_{2} \ldots a_{k}$ a partial valid execution se- 
quence, I a set of indices such that $i \in I$ iff $\hat{Q}_{i}(k)>0$. Suppose that a subset $I^{\prime} \subset I$ exists such that $\hat{q}_{i}-\hat{r}_{i} /$ fo for each $i \in I^{\prime}$. For each $i \in I^{\prime}$ substitute $\hat{r}_{i}$ with a new vector $\hat{r}_{i}^{\prime}=\left(r_{i}^{\prime}=\left(r_{i}^{\prime}, r_{i 2}^{\prime}, \ldots, r_{i m}^{\prime}\right)\right.$ such that $r_{i j}^{\prime}=r_{i j}$ if $q_{i j} \geqslant r_{i j}$, and $r_{i j}^{\prime}=q_{i j}$ if $\hat{q}_{i j}<\hat{r}_{i j}$. Then lemma 1 can be applied but conditions (4.1) and (4.2) are never necessary.

Suppose now that a task system $G$ can be executed level by level, that is a valid execution sequence $\alpha^{*}=a_{1}^{*} a_{2}^{*} \ldots a_{2 n}^{*}$ exists such that if $a_{i}^{*}=I, a_{j}^{*}=\bar{T}^{\prime}$ and $\ell(T)>\ell\left(T^{\prime}\right)$, then $i<j$. Let $\alpha=a_{1} a_{2} \ldots a_{k}$ be any valid partial execution sequence, $s^{\prime}$ a set of integers such that $s \in S^{\prime}$ iff:
1. $\exists i: \bar{T}_{i} \in \alpha \quad \ell\left(T_{i}\right)=s$, and
2. $\exists j: \overline{\mathrm{T}}_{j} \notin \alpha$ and $\ell\left(\mathrm{T}_{j}\right)=\mathrm{s}$.

and $S$ a set of integers such that $s \in S$ iff $\min \left\{S^{\prime}\right\} \leqslant s \leqslant \max \left\{S^{\prime}\right\}$. For each $s \in S$ let $I_{s}$ be a set of indices such that $i \in I_{s}$ iff $\bar{T}_{i} \notin \alpha$ and $\ell\left(T_{i}\right)=s$. Modify every vector $\hat{r}_{i}, \forall i \in I_{s}, \forall s \in S$, according to the assumptions of corollary 1 , if $\hat{q}_{i}-\hat{r}_{i} 0$. Let $I_{s p}$ be the subset of $I_{s}$ such that $i \in I_{s p}$ iff $\hat{q}_{i}-\hat{r}_{i}<0$, and $I_{s n}$ be the subset of $I_{s}$ such that $i \in I_{\text {in }}$ iff $\hat{q}_{i}-\hat{r}_{i}>0$. Under these assumptions, the following basic theorem can be proved:

Theorem 4: For every $s \in S$, if no set of indices $D_{s p} \in I_{s p}$ exists, such that, for each $i \in D_{S p}$ :

$$
\hat{q}_{i} * \hat{v}(k)-\sum_{s^{\prime} \in S} \sum_{t \in I_{s^{\prime}}}\left(\hat{q}_{t}-\hat{r}_{t}\right)+\sum_{\substack{j \in I_{s p} \\ j \notin D_{s p}}}\left(\hat{r}_{j}-\hat{q}_{j^{\prime}}\right)
$$

and no set of indices $D_{s n} \subset I_{s n}$ exists, such that, for each $i \in D_{s n}$ :

$$
\hat{r}_{i} \neq \hat{v}(k)-\sum_{\substack{s^{\prime} \in S \\ s^{\prime}>s}} \sum_{t \in I_{s^{\prime}}}\left(\hat{q}_{t}-\hat{r}_{t}\right)+\sum_{j \in I_{s p}}\left(\hat{r}_{j}-\hat{q}_{j}\right)+\sum_{j \in D}\left(\hat{r}_{j}-\hat{q}_{j}\right)
$$

then the state $s_{k}$ is safe.

Proof. Let $s_{\max }, s_{\min }$ be respectively the maximum and minimum of set $\mathrm{s}$. By assumption, for every $i \in \mathrm{I}_{\mathrm{s}_{\max }}$, if $\mathrm{T}_{j} \ll \mathrm{T}_{i}$, then $\overline{\mathrm{T}}_{j} \in \alpha$; therefore task $\mathrm{T}_{i}$ is ready to be initiated or will be ready after a finite time interval. In any case, the initiation of $\mathrm{T}_{i}$ is constrained only by the availability of resources. But, by lema 1 , if conditions (4.3) and (4.4) axe satisfied, a partial valid execution sequence $\alpha_{s_{\max }}$ can be found, such that $I_{i} \in \alpha_{s_{\max }}$ for every $i \in I_{s_{\max }}$. Hence a11 tasks at level $s_{\max }-1$ will be 
ready for initiation after a finite time form event $a_{k}$. But, by lema 1 , if conditions (4.3) and (4.4) are satisfied, a partial valid execution sequence $\alpha s_{\max }-1$ can be found, such that $\underline{T}_{i} \in \alpha_{\mathrm{s}_{\max }-1}$ for every $i \in \mathrm{I}_{\mathrm{S}_{\max }-1}$, and so on. Therefore, if conditions (4.3) and (4.4) are satisfied, all task at a level $s \geqslant s_{\min }$ can be completed with the currently available resources; let $\alpha_{1}=\alpha_{s_{\max }}\left\|\alpha_{s_{\max }-1}\right\| \ldots \| \alpha_{s_{\text {min }}}$ be the valid partial completion sequence containing the terminations of all tasks $\mathrm{I}_{i}$, for $i \in \bigcup_{S \in S} I_{S}$. Consider now the partial execution sequence $\alpha_{2}$ containing all and only all the terminations of al1 task $T$ such that $\bar{T} \in \alpha$ but $\underline{T} \& \alpha$; a termination event does not decrease the amount of available resources, and therefore a new valid partial execution sequence $\alpha^{\prime}$ can be built merging $\alpha_{1}$ and $\alpha_{2}$. If $\alpha_{s_{\min }}^{*}$ is the prefix of the valid execution sequence $\alpha^{*}$ such that $\underline{T} \epsilon \alpha_{s_{\min }}^{*}$ for every $T$ such that $l(T) \geqslant$ $\geqslant s_{\min }$ and $T^{\prime} \phi \alpha_{s_{\min }}^{*}$ for every $T^{\prime}$ such that $\ell\left(T^{\prime}\right)<s_{\min }$, then the two sequences $\alpha \| \alpha^{\prime}$ and $\alpha_{s_{\text {min }}}^{*}$ contain exactly the same events; therefore, if $\alpha^{\prime *}$ in the completion sequence such that $\alpha^{*}=\alpha_{s_{\min }}^{*} \| \alpha^{* *}$, then $\alpha^{\prime *}$ is also a valid completion sequence for the partial execution sequence $\alpha \| \alpha^{\prime}$, that is $\alpha\left\|\alpha^{\prime}\right\| \alpha^{{ }^{*}}$ is a valid execution sequence, and consequentiy the state $s_{k}$ is safe.

The last problem is to find sets $D_{p}$ and $D_{n}$ in lemma $1, D$ in theorems $I$ and 2 , and $D_{s p}$ and $D_{s n}$ in theorem 4 . This problem is easily solved because:

1. All sets can be searched independently of each other.

2. The search always involves a set of tasks which, upon termination, do not increase (decrease) the amount of available resources; therefore the order of allowable initiation events is not important [2].

Then the simplest algorithm for the detection of sets $D_{p}$ and $D_{n}$ in lemma 1 is the following:

\section{A1gorithm 1:}

Step 1: detection of set $D_{p}$

1. Initialize $D_{p}=I_{p}$ and $\hat{V}-\hat{v}(k)$.

2. Search for any index $i \in I_{p}$ such that $\hat{q}_{\hat{i}} \leqslant \hat{v}$; if none is found goto 4 .

3. $\mathrm{D}_{\mathrm{p}} \leftarrow \mathrm{D}_{\mathrm{p}}-\{\mathrm{i}\} ; \hat{\mathrm{V}} \leftarrow \hat{\mathrm{V}}+\left(\hat{\mathrm{r}}_{\mathrm{i}}-\hat{\mathrm{q}}_{\mathrm{i}}\right) ;$ goto 2 .

Step 2: detection of set $D_{n}$

4. Initialize $D_{n}=I_{n}$ and $\hat{v}-\hat{v}+\sum_{j \in I_{n}}\left(\hat{r}_{j}-\hat{q}_{j}\right)$.

5. Search for any index $i \in I_{n}$ such that $\hat{r}_{i} \hat{v}$; if none is found terminate the algorithm.

6. $D_{n}-D_{n}-|i| ; \hat{v}-\hat{V}+\left(\hat{q}_{i}-\hat{r}_{i}\right) ;$ goto 5 .

If the number of tasks in $I_{p} \cup I_{n}$ is $z$, the running time of the algorithm is $O\left(m \cdot z^{2}\right)$. Using more sophisticated techniques, it is possible to speed up the algorithn, reaching 
a running time of $O(\mathrm{~m} \cdot \mathrm{z}) \quad[2]$. Sets $\mathrm{D}_{\mathrm{sp}}$ and $\mathrm{D}_{\mathrm{sn}}$ in theorem 4 , and set $\mathrm{D}$ in theorems 1 and 2 can be found in a very similar fashion.

\section{Conclusions}

The formulation of the deadlock detection and avoidance problem made by Coffman and Denning has been extended here to cover the more general case of a graph structured task system. The proposed approach preserves the basic property of the simpler models developed for independent chains of tasks, i.e. it allows the definition of non-enumerative avoidance algorithms. The conditions on which such algorithms are based are only sufficient, and therefore it can happen that a resource request is not accepted even if it could be granted without entering an unsafe state; however, without this approximation, we are unable to design algorithms which are not essentially enumerative.

\section{APPENDIX}

Proof of theorem 1. Suppose that at least one index i $\epsilon D$ exists such that it is possible to find a partial valid execution sequence $\alpha^{\prime}=a_{1} a_{2} \ldots a_{k} a_{k+1} \ldots a_{p}$, $\alpha$ being a prefix of $\alpha^{\prime}$ and $a_{p}=\bar{T}_{i}$. Let the sequence $\alpha^{\prime \prime}=a_{k+1} \ldots a_{p}$ be a subset of $\alpha^{\prime}$. Suppose also that $\alpha^{\prime \prime}$ does not contain the initiation of any task $T_{j}, j \in D$. If this is not the case, and a set of events $\left\{a_{k_{1}}, a_{k_{2}}, \ldots, a_{k_{p}}\right\}$ exists such that $a_{k_{s}} \epsilon \alpha^{\prime \prime}$ and $a_{k_{s}}=\bar{T}_{i_{s}}$ for $i_{s} \in D, \quad 1 \leqslant s \leqslant p$, it is always possible to impose new values to $p$ and $i$ such that $a_{k}=a_{k_{1}}, i=i_{1}$ and $\alpha^{\prime \prime}=a_{k+1} \ldots a_{k_{1}}-1$. Task $T_{i}$ is initiated, hence $\hat{Q}_{i}(p-1) \leqslant \hat{v}(p-1)$. Let $A$ be the set of indices such that $j \varepsilon A$ iff $\alpha$ " contains the event $\overline{\mathrm{T}}_{j}$, and $\mathrm{B}$ the set of indices such that $j \in \mathrm{B}$ iff $\alpha^{\prime \prime}$ contains the event $\underline{T}_{j}$; then:

$$
\begin{aligned}
& \hat{\mathrm{v}}(\mathrm{p}-1)=\hat{\mathrm{v}}(\mathrm{k})+\sum_{j \in \mathrm{B}} \hat{\mathrm{r}}_{\mathrm{j}}-\sum_{\ell \in \mathrm{A}} \hat{\mathrm{q}}_{\ell}= \\
& =\hat{v}(k)+\sum_{j \in B^{\top}} \hat{r}_{j}+\sum_{\ell \in B \wedge A} \hat{r}-\sum_{u \in A^{\top}} \hat{q}_{u}-\sum_{s \in B \wedge A} \hat{q}_{s}= \\
& =\hat{v}(k)+\sum_{j \in B^{r}} \hat{r}_{j}-\sum_{u \in A^{\top}} \hat{q}_{u}+\sum_{l \in B \wedge A}\left(\hat{r}_{\ell}-\hat{q}_{\ell}\right)
\end{aligned}
$$

being $B^{\prime}=B \wedge(B \wedge A)$ and $A^{\prime}=A \wedge(\overline{B A A})$. If $\hat{P}_{\ell \text {, fin }}$ is a vector specifying the total amount of resources held by task $\mathrm{T}_{\ell}$ after its initiation (and before its termination), then we can write:

$$
\begin{aligned}
& \hat{v}(p-1) \leqslant \hat{v}(k)+\sum_{j \in B^{\top}} \hat{r}_{j}+\sum_{l \in B \wedge A}\left(\hat{r}_{\ell}+\hat{p}_{\ell}(k)+\sum_{s \in B} \hat{t}_{s l}-\hat{p}_{\ell, f i n}\right)= \\
= & \hat{v}(k)+\sum_{j \in B}\left(r_{j}+\sum_{s \in B \wedge A} \hat{t}_{j s}\right)+\sum_{l \in B \wedge A}\left(\hat{x}+\sum_{s \in B \wedge A} \hat{t}_{s \ell}+\right. \\
+ & \hat{P}_{\ell}(k)-\hat{p}_{\ell, f_{i n}} \leqslant
\end{aligned}
$$




$$
\leqslant \hat{v}(k)+\sum_{j \in B^{\top}} \hat{p}_{j}(k)+\sum_{l \in B \wedge A} \hat{p}_{\ell}(k)=\hat{v}(k)+\sum_{j \in B} \hat{p}_{j}(k)
$$

Therefore it can be stated that:

$$
\hat{Q}_{i}(k)=\hat{Q}_{i}(p-1) \leqslant \hat{v}(k)+\sum_{j \in B} \hat{p}_{j}(k)
$$

By definition $B \wedge D=\emptyset$, and consequently $B \wedge D^{*}=\emptyset$; hence $\left.\hat{Q}_{i}(k) \hat{v}^{\prime} k\right)+\sum_{j \in D \wedge D} \hat{p}_{j}(k)$, which contradicts the theorem.

So far we have proved that (2.2) is a sufficient condition for deadlock detection. We will now prove that it is also necessary. Let $D$ be a set of indices such that $i \in D$ iff no valid partial execution sequence $\alpha_{i}$ can be found leading to initiation of $T_{j}$ and having, as a prefix, $\alpha$ (every task $\mathrm{T}_{i}, i \in \mathrm{D}$, is deadlocked). Then, in the best case, it is possible to find a partial valid execution sequence having $\alpha$ as a prefix and containing the termination of all tasks $T_{j}$, $j \notin D v D^{*}$. Let $\alpha^{\prime}=a_{1} a_{2} \ldots a_{k} a_{k+1} \ldots a_{p}$ be such a sequence. Being $T_{i}, \nvdash i \in D$, a deadlocked task, $\hat{Q}_{i}(p) \leqslant \hat{v}(p)$, and no task $T_{j}$ exists such that $\hat{Q}_{j}(p) \leqslant \hat{v}(p)$, because only tasks $T_{i}$, $i \in D$, are asking for resources in state $s_{p}$. Therefore set $D$ satisfies condition (2.2). This means that, if state $s_{k}$ contains a deadlock, a state $s_{p}, p \geqslant k$, will be necessarily found, in which condition $(2.2)$ is satisfied.

\section{References}

Coffman, E.G.jr., Elphick, M.J., Shoshani, A. "System Deadlocks", Computing Surveys 2 , (1971), 67-78.

Coffman E.G.jr., Denning, P.J. "Operating Systems Theory", Prentice Ha11, 1973.

Habermann, A.N. "Prevention of System Deadlock", Corm. ACM 12, (1969), 373-377.

Havender, J.W. "Avoiding Deadlock in Multitasking Systems", IBM Syst. J. 7, (1968), 74-84.

Hebalkar, P.G. "A Graph Model for Analysis of Deadlock Prevention in Systems with Parallel Computation" , Proceed. IFIP Congress (1971), 168-172.

Howard, J.H.jr. "Mixed Solutions for Deadlock Problem", Comm.ACM 16, (1973), 427-430.

Llewellyn, J.A. "The Dead1y Embrace - a Finite State Model Approach", Computer Journa1 $16,(1973), 223-225$. 